\title{
Wessen Interessen vertritt die AGZ eigentlich?
}

Für den Vorstand der AGZ: W. Grete

Seit vielen Jahren hinterfragt Kollege Tapernoux die ärztliche Standespolitik regelmässig mit recht hemdsärmligen Rundumschlägen. Wir sind ihm dafür Dank schuldig, zwingen doch seine Anmerkungen zur Reflexion.

Der Vorstand der Zürcher Ärztegesellschaft bereitet als Exekutive Geschäfte vor, unterbreitet diese der Delegiertenversammlung und versucht anschliessend, deren Beschlüsse umzusetzen. Die breite Mitgliederumfrage «Arzt 2000» zeigte, dass die grosse Mehrheit unserer sehr heterogenen Gesellschaft diese Politik trägt. Es sei aber die Frage gestattet, ob die Interessen von Herrn Tapernoux tatsächlich die Interessen der Zürcher Ärzteschaft sind.

Im Vorfeld wichtiger Entscheidungen sollen aber auch unkonventionelle Argumente in die Diskussion einfliessen können. Nur dergestalt kann die Befindlichkeit eines sehr inhomogenen Berufsverbandes eruiert werden. Als Präsident der AGZ danke ich deshalb der Redaktion der Schweizerischen Ärztezeitung für dieses Forum.

\section{Die ärztliche Betreuung von Asylbewerbern}

Gemäss Bundesgesetz können Versicherungen mit eingeschränktem Zugang zu Leistungserbringern für Asylbewerber etabliert werden. Im Kanton Zürich wurde vom Departement für soziale Sicherheit mit der Helsana ein derartiges Modell ausgearbeitet und präsentiert. Damit nicht der Staat oder die Krankenversicherer willkürlich Leistungserbringer aussuchten oder gar einsetzten, hat die AGZ interveniert und mittels einer Umfrage geklärt, wer allenfalls bereit sei, Asylbewerber ärztlich zu betreuen. Dies war der Ursprung einer Liste interessierter Ärztinnen und Ärzte, welche sich gemäss eigener Meldung besonders mit Problemen der Migrationsmedizin auseinandersetzen wollten. Herr Tapernoux unterstellt nun unreflektiert der AGZ, sie hätte bei der Auswahl mitgewirkt. Dies hat die AGZ nie getan, sondern sie hat sich immer dafür eingesetzt, dass alle interessierten Kolleginnen und Kollegen berechtigt blieben, Patientinnen und Patienten unabhängig von Herkunft und Versicherungsstatus weiter zu betreuen.
Die AGZ hat aber Hand geboten, zusammen mit der Justizdirektion, der Helsana und den lokalen Behörden, dafür zu sorgen, dass Asylbewerber in der Gesundheitsversorgung nicht zum Spielball von divergierenden, politischen Interessen werden.

\section{Die Diskussion um den Kontrahierungs- zwang}

Die Aufhebung des Kontrahierungszwangs wäre in der Tat ein fundamentaler Systemwechsel in unserem Gesundheitswesen. Die nackte Drohung mit dem Referendum hingegen ist für den Vorstand der AGZ und insbesondere für deren Präsidenten nun aber wirklich keine intelligente Option. Glaubt denn Kollega Tapernoux tatsächlich, dass ein Referendum zur Vertragsfreiheit eine Lösung der Probleme bringen würde? Erstens dürfte es sehr, sehr schwierig werden, eine Referendumsabstimmung zu gewinnen, wenn landauf und landab mit günstigeren Kassenprämien gelockt würde und gleichzeitig der Bevölkerung versprochen würde, dass $95 \%$ aller Leistungserbringer Vertragspartner bleiben. Die ärztliche Opposition würde als Schutz des Berufsverbandes für schwarze Schafe desavouiert. Die Diskussion im Ständerat sollte uns warnen.

Aber auch ein Abstimmungserfolg mit «Nein» würde einen Scherbenhaufen hinterlassen und Reformen auf Jahre blockieren. In dieser Zeit würde sich die Spirale - mehr Leistungserbringer (das sind nicht nur Ärztinnen und Ärzte und sie kommen nun auch aus Nachbarländern ) - mehr Leistungen - mehr eingesetzte Zeit am Krankenbett - mehr Kosten, aber stagnierende Taxpunktwerte - weiterdrehen. Dieser Prozess führt zwingend zu einer Verarmung und Qualitätsgefährdung der ambulanten Medizin. Seit 10 Jahren haben alle Kantone massiv an Taxpunktwertsubstanz verloren und die Leistungserbringer kompensieren - soweit möglich - durch Mehrleistungen. Daran wird auch TARMED nichts ändern.

Alternativen sind somit gefragt, z.B. eine einfache Grundversicherung mit eingeschränkter Arzt- und Spitalwahl. Dazu die grosse, einfache Zusatzversicherung «alle Ärzte». 50 bis $60 \%$ der
Ärztegesellschaft des Kantons Zürich Freiestrasse 138 CH-8032 Zürich 
Bevölkerung würden sich dort versichern. Damit wäre der ambulante «Privatpatient» wieder geboren. Jeder Arzt mit Berufsbewilligung darf ihn betreuen. Das würde der Bevölkerung und der Ärzteschaft die verlorene Freiheit im ambulanten Bereich teilweise wieder zurückgeben.

Versuchen wir deshalb aktiv und nicht rein defensiv, Vorschläge einzubringen und gemeinsam an Verbesserungen zu arbeiten und nicht nur «njet» zu sagen, bis uns die Zeit restlos überholt hat. Mehr dazu auf der Homepage der AGZ www.aerzte-zh.ch. Wir sind uns aber bewusst, dass hier die Diskussion noch geführt werden muss und noch alle Optionen offen sind. Die Mehrheit der Zürcher Ärztinnen und Ärzte verwirft heute jede Lockerung des Vertragszwangs.

\section{Notfalldienstreglement}

In der Regelung des Notfalldienstes sind die Bezirksärztegesellschaften frei. Subsidiär hat die AGZ bei Streitigkeiten und Missständen einzugreifen. Unser Reglement dient einzig und allein dieser schiedsrichterlichen Funktion. Es wurde der Delegiertenversammlung vorgelegt und angenommen. Bis heute war die Unabhängigkeit der Bezirksgesellschaften bei der Organisation des Notfalldienstes ein berechtigtes Anliegen, lassen sich doch die Verhältnisse zwischen Paradeplatz und dem Hörnli nicht immer über einen Leisten zu schlagen. Erstaunt bin ich über den Ruf nach dem Staat bei der Lösung von Organisation und Inkasso bei Notfalldienstleistungen. Ich möchte hier nachdrücklich vor ärztlichem Druck auf die Gemeinden warnen, ist doch ein flächendeckender Notfalldienst über Spitalambulatorien oder Notfalldienstlisten aus den Gemeindekanzleien kaum das Herzensanliegen von Herrn Kollegen Tapernoux. In vielen Notfallregionen finanzieren die Gemeinden übrigens die Information der Bevölkerung.

\section{Neues Patientenrecht im Kanton Zürich}

Innerhalb der kantonalen Ärztegesellschaft wurde zum Patientenrechtsgesetz eine Vernehmlassung durchgeführt. Der vorliegende Entwurf wurde von der Öffentlichkeit und den interessierten Kreisen sehr gut aufgenommen. Die AGZ hat bereits im Vorfeld aktiv mitgearbeitet. Wir sind mit Kollega Tapernoux einverstanden, dass das Patientengeheimnis zwar Leitgedanke bleiben muss, aber eine vernünftige Kommunikation unter den beteiligten Kolleginnen und Kollegen zugunsten der Kranken erhalten bleiben muss und begrüssen deshalb auch die Ergänzungen von lic. iur. Oskar Lehmann.

ICD-10 wird an den Zürcher Spitälern seit Jahren verwendet. Persönlich bin ich der Ansicht, dass nur exakte Diagnosen (verschlüsselt) die Leistungen im Gesundheitswesen vergleichbar machen. Es ist scheinheilig, sich gegen exakte, vergleichbare Diagnosen (auch in der ambulanten Medizin) zu wehren und gleichzeitig ein Qualitätsund Kostenmanagement zu fordern oder gar Kollegen wegen hoher Rechnungen zu kritisieren, ohne das Fundament zu definieren. Transparenz dient hier vor allem den ärztlichen Leistungserbringern.

\section{«Swica Nova light»-Modell}

Herrn Kollegen Tapernoux ist entgangen, dass die Wettbewerbskommission der AGZ bei Strafandrohung verboten hat, in dieser Angelegenheit weiter aktiv zu sein. Wir haben dies eingehend kommuniziert. Die Aktion zeigte nachhaltige Wirkung und hat zumindest verhindert, dass andere Versicherungen dem Beispiel der SWICA leichtfüssig gefolgt sind. Die Solidarität der Zürcher Ärzteschaft hat mich tief beeindruckt.

(Ich bin nicht einmal sicher, ob mit der Aussage, dass sich jeder einzelne Arzt weiterhin gegen die Aufnahme auf die Liste wehren darf und soll, nicht schon wieder ein Verfahren der WEKO provoziert wird. PS. Die Swica hat das Modell übrigens umgetauft. Seit dem Vergleich mit der AGZ macht die Versicherung in dieser Sache auch keine öffentliche Werbung mehr.)

Abschliessend möchte ich dazu aufrufen, die Standespolitik nicht als eine Abwehrschlacht gegen böse Versicherungen, Behörden und obskure Alternativen und Autoritäten zu gestalten.

Gemeinsam Erarbeitetes hat Bestand. Die Ärzteschaft hat einst die Einrichtung von Krankenkassen bekämpft! Nicht auszudenken, was geschehen wäre, wenn dieses Ansinnen gelungen wäre. Bedenken wir, dass es nicht mehr genügt, wenn der Kranke mit seinem Arzt zufrieden ist; auch die mitfinanzierende Solidargemeinschaft muss mit dem System zufrieden sein. Daran lasst uns arbeiten. 